J. Korean Soc. Math. Educ. Ser. B: Pure Appl. Math.

ISSN(Print) 1226-0657

http://dx.doi.org/10.7468/jksmeb.2012.19.4.349

ISSN(Online) 2287-6081

Volume 19, Number 4 (November 2012), Pages 349-361

\title{
GROUPS ACTING ON MEDIAN GRAPHS AND MEDIAN COMPLEXES
}

\author{
DOHYOUNG RYANG
}

\begin{abstract}
CAT(0) cubical complexes are a key to formulate geodesic spaces with nonpositive curvatures. The paper discusses the median structure of CAT(0) cubical complexes. Especially, the underlying graph of a CAT(0) cubical complex is a median graph. Using the idea of median structure, this paper shows that groups acting on median complexes are $\mathrm{L}(\delta)$ groups and, in addition, word $\mathrm{L}(0)$ groups are closed under taking free product.
\end{abstract}

\section{INTRODUCTION}

A finitely generated group can be studied, in geometric view points, by investigating a metric space which the group acts on. If a group $G$ acts properly, cocompactly, and by isometries on a metric space $X$, then we call $G$ acts geometrically on $X$. Throughout this paper, a group is finitely generated and a group action is a geometric action in case not mentioned. For example, hyperbolic groups are the groups acting on hyperbolic spaces. This group has been well researched since Gromov [6]. Then, $\mathrm{L}(\delta)$ geodesic spaces for a nonnegative constant $\delta$ were introduced as a generalization of hyperbolic spaces. Mathematicians has been more interested in $\mathrm{L}(0)$ spaces, because the 0 -skeleton of a $\operatorname{CAT}(0)$ cubical complex has the $\mathrm{L}(0)$ property.

The CAT(0) cubical complex provides a key idea to formulate geodesic spaces of nonpositive curvatures. Gromov [6] showed that CAT(0) cubical complexes can be characterized in a combinatorial way though which the space can be studied in geometrical points of view. See [1] for related results and application. Properties of CAT(0) complexes has been found related to the associated groups. Sageev

Received by the editors July 12, 2012. Accepted October 19, 2012.

2010 Mathematics Subject Classification. 20F65.

Key words and phrases. CAT(0) cubical complex, free product, L(0) space, median graph. 
presented further properties of CAT(0) cubical complexes about the ends of groups [12]. Niblo and Reeves found some properties about groups acting on CAT(0) cubical complexes: There is a way to find a bicombing of Caley graphs of CAT(0) complexes' fundamental groups [9]; groups with Kazhdan's property (T) have no unbounded actions on finite dimensional $\mathrm{CAT}(0)$ cubical complexs [8]; Coxeter groups act on CAT(0) cubical complexes [10].

This paper discusses that the underlying graph of a CAT(0) cubical complex is a median graph. Conversely, a CAT(0) cubical complex is constructible from a given median graph. Thus, CAT(0) cubical complexes are median complexes on which then $\mathrm{L}(\delta)$ property is introduced. A retraction $r$ from a CAT $(0)$ cubical complex to its underlying graph is a quasi-isometry so the $\mathrm{L}(\delta)$ property is preserved under $r$. Using these ideas, as main results, Theorems 4.2 and 4.4 are show that finitely generated groups acting on an $n$-median complexes are $\mathrm{L}(\delta)$ groups; a group acting on a median graph is an $\mathrm{L}(0)$ group and is closed under taking free product.

\section{Preliminaries}

2.1. Metric graphs. Let $(X, d)$ be a metric space. A path is a mapping from a segment $[a, b]$ to $X$. A geodesic joining two points $x, y \in X$ is a mapping $g$ : $[a, b] \rightarrow X$ such that $g(a)=x, g(b)=y$ and $d\left(g(t), g\left(t^{\prime}\right)\right)=\left|t-t^{\prime}\right|$ for all $t, t^{\prime} \in$ $[a, b]$. A geodesic segment $[x, y]$ is the image of the geodesic $g:[a, b] \rightarrow X$. A geodesic metric space is a metric space in which every pair of points can be joined by a geodesic segment. Let $(X, d)$ and $\left(Y, d^{\prime}\right)$ be metric spaces. Then, $(X, d)$ is isometrically embedded into $\left(Y, d^{\prime}\right)$ if there is a mapping $f: X \rightarrow Y$ such that $d(x, y)=d^{\prime}(f(x), f(y))$ for all $x, y \in X$. In this case, $X$ is a subspace of $Y$, and $Y$ is an extension of $X$. A mapping $r: Y \rightarrow X$ is a retraction if $r$ is idempotent nonexpansive, i.e., $r(x)=x$ for all $x \in X$ and $d^{\prime}\left(x^{\prime}, y^{\prime}\right) \geq d(x, y)$ for any $x^{\prime}, y^{\prime} \in Y$ where $x=r\left(x^{\prime}\right), y=r\left(y^{\prime}\right)$. The image $r(Y)$ is called a retract of $Y$.

A graph $\Gamma$ is the pair $(V, E)$ of vertices $V$ and edges $E$ joining vertices. Define the distance $d(x, y)$ between any $x, y \in V$ as the length of a shortest path joining $x$ and $y$. Now $(\Gamma, d)$ is a metric graph. An interval $I[x, y]$ in $\Gamma$ is the set of all points on a shortest path between the two points $x$ and $y$, i.e.,

$$
I[x, y]=\{z \in \Gamma \mid d(x, z)+d(z, y)=d(x, y)\} .
$$

Three vertices $x, y$, and $z$ in $\Gamma$ form a metric trianlge $x y z$ if the intervals $I[x, y]$, $I[y, z]$, and $I[z, x]$ pairwise intersect only in common end vertices. Four vertices $x, y$, 
$z$, and $v$ form a metric rectangle $x y z v$ if $x, z \in I[y, v], y, v \in I[x, y]$, and the opposite sides have the same length. Note that metric triangles and metric rectangles need not to be unique.

A subgraph $\Omega$ is said to be gated in $\Gamma$ provided there exists a vertex $y^{\prime} \in \Omega$ such that each vertex $y \in \Omega$ is connected to all $x \in \Gamma-\Omega$ by a shortest path via $y^{\prime}$. Such $y^{\prime}$ is called the gate for $x$ into $\Omega$. A graph $\Gamma$ is a gated amalgam of two graphs $\Gamma_{1}$ and $\Gamma_{2}$ if $\Gamma_{1}$ and $\Gamma_{2}$ constitute two intersecting gated subgraphs of $\Gamma$ whose union is the whole $\Gamma$.

The Catesian product $\Gamma=\Gamma_{1} \times \cdots \times \Gamma_{n}$ of graphs $\Gamma_{1}, \ldots, \Gamma_{n}$ is the graph whose vertices are the $n$-tuples $\left(x_{1}, \ldots, x_{n}\right)$ with $x_{i}$ from $\Gamma_{i} . \Gamma$ has an edge between two vertices $x=\left(x_{1}, \ldots, x_{n}\right)$ and $y=\left(y_{1}, \ldots, y_{n}\right)$ if and only if $x_{i}$ and $y_{i}$ are adjacent in $\Gamma_{i}$ for some $i$ and $x_{j}=y_{j}$ for other all $j \neq i$. The distance between $x$ and $y$ is

$$
d_{\Gamma}(x, y)=\sum_{i=1}^{n} d_{\Gamma_{i}}\left(x_{i}, y_{i}\right) .
$$

The $n$-hypercube $H_{n}$ is the Cartesian product of $n$ copies of the 1-dimensional cube.

2.2. CAT(0) cubical complexes. A cubical complex is a collection of cubes of any dimensions which is closed under taking subcells and nonempty intersections. Cubes of a cubical complex are called faces. See Bridson and Haefliger [1] for details. Let $K$ be an abstract cubical complex. The vertex set $V$ is the set of all 0 -dimensional faces of $K$, and the edge set $E$ is the set of all 1-dimensional faces of $K$. The pair $(V, E)$ is called the 1-skeleton, denoted by $K^{(1)}$, or the underlying graph of $K$. Conversely, from a graph $\Gamma$, we can construct a cubical complex $K(\Gamma)$ by taking all induced subhypercubes as faces of the complexes.

An abstract cubical complex and its realization are identical. The geometric realization $|K|$ of a cubical complex $K$ is the polyhedral complex obtained by replacing every face $\sigma$ by a solid unit cube $|\sigma|$ of the same dimension such that realization commutes with intersection, that is, $\left|\sigma^{\prime}\right| \cap\left|\sigma^{\prime \prime}\right|=\left|\sigma^{\prime} \cap \sigma^{\prime \prime}\right|$ for any two faces $\sigma^{\prime}$ and $\sigma^{\prime \prime}$. Obviously, $|K|=\cup\{|\sigma|: \sigma \in K\}$. Analogously, for a planar graph $\Gamma$, the geometric realization $|\Gamma|$ is a polygonal complex by replacing each inner face with $k$ sides of $\Gamma$ by a regular $k$-gon with side length 1 in the Euclidean plane.

The geometric realization $|K|$ of a complex $K$ can be endowed with an intrinsic path metric. Inside a maximal face $|\sigma|$ of $|K|$, the distance is measured by $\ell_{1}$ taxi-cab metric. For any two points $x, y \in|K|$, the distance $d(x, y)$ is defined by the greatest lower bound on the lengths of the paths joining $x$ and $y$. A path in $|K|$ from $x$ to 
$y$ is a sequence $x=x_{0}, x_{1}, \ldots, x_{m}=y$ such that there exists a face $\left|\sigma_{i}\right|$ containing $x_{i}$ and $x_{i+1}$, and the length of the path equals $\sum_{i=0}^{n-1} \ell_{1}\left(x_{i}, x_{i+1}\right)$, where $\ell_{1}\left(x_{i}, x_{i+1}\right)$ is computed inside $\left|\sigma_{i}\right|$ according to the respective metric. Then, $|K|$ becomes an geodesic metric space. A geodesic joining $x$ and $y$ in $|K|$ is a maping $\gamma:[a, b] \rightarrow|K|$ such that $|b-a|=d(x, y), \gamma(a)=x, \gamma(b)=y$, and $d(\gamma(s), \gamma(t))=|s-t|$ for all $s, t \in[a, b]$.

The CAT(0) property handles the metric space with non-positive curvatures. A geodesic triangle $\Delta\left(x_{1}, x_{2}, x_{3}\right)$ in a geodesic metric space $(X, d)$ consists of three points in $X$ (which is called vertices of $\Delta$ ) and a geodesic segments between each pair of vertices (which is called edges of $\Delta$ ). The triangle $\Delta$ need not to be unique. A comparison triangle for $\Delta\left(x_{1}, x_{2}, x_{3}\right)$ is the triangle $\Delta\left(x_{1}^{\prime}, x_{2}^{\prime}, x_{3}^{\prime}\right)$ in $\mathbb{E}^{2}$ such that $d\left(x_{i}, x_{j}\right)=d_{\mathbb{E}^{2}}\left(x_{i}^{\prime}, x_{j}^{\prime}\right)$ for $i, j \in\{1,2,3\}$ and $j \equiv i+1 \bmod 3$. A geodesic metric space $X$ to be a $\operatorname{CAT}(0)$ space provided all geodesic triangles in $X$ satisfy the comparison axiom below. There are many results and applications about CAT(0) spaces and CAT(0) cubical complexes. See Chepoi [2] for more detailed description.

Axiom 2.1 (Cartan-Alexandrov-Toponogov). Let $(X, d)$ be a geodesic space and let $\Delta\left(x_{1}, x_{2}, x_{3}\right)$ be a geodesic triangle in $X$. Let $y$ be a point on the geodesic joining $x_{1}$ and $x_{2}$. If $y^{\prime}$ denote a unique point on the line segment $\left[x_{1}^{\prime}, x_{2}^{\prime}\right]$ of the comparison triangle $\Delta\left(x_{1}^{\prime}, x_{2}^{\prime}, x_{3}^{\prime}\right) \in \mathbb{E}^{2}$ such that $d\left(x_{i}, y\right)=d_{\mathbb{E}^{2}}\left(x_{i}^{\prime}, y^{\prime}\right)$ for $i=1,2$, then

$$
d\left(x_{3}, y\right) \leq d_{\mathbb{E}^{2}}\left(x_{3}^{\prime}, y^{\prime}\right) .
$$

2.3. Quasi-isometry. The idea of quasi-isometry is to see two geodesic spaces to be equal on a large scale. Let $(X, d)$ and $\left(X^{\prime}, d^{\prime}\right)$ be geodesic spaces and let $\lambda \geq 1$ and $\varepsilon \geq 0$ be constants. A mapping $f:(X, d) \rightarrow\left(X^{\prime}, d^{\prime}\right)$ is said to be a $(\lambda, \varepsilon)$-quasiisometric embedding if

$$
\frac{1}{\lambda} d(x, y)-\varepsilon \leq d^{\prime}(f(x), f(y)) \leq \lambda d(x, y)+\varepsilon
$$

for all $x, y \in X$. In addition, $f$ is called a $(\lambda, \varepsilon)$-quasi-isometry if there exists a constant $C \geq 0$ such that every point in $X^{\prime}$ lies in the $C$-neighborhood of the image of $f .(X, d)$ and $\left(X^{\prime}, d^{\prime}\right)$ are said to be quasi-isometric when such $f$ exists.

If $f:(X, d) \rightarrow\left(X^{\prime}, d^{\prime}\right)$ is a $(\lambda, \varepsilon)$-quasi-isometry, then there exist a constant $C \geq 0$ and a $\left(\lambda^{\prime}, \varepsilon^{\prime}\right)$-quasi-isometry $g: X^{\prime} \rightarrow X$ for some $\lambda^{\prime} \geq 1$ and $\varepsilon^{\prime} \geq 0$ such that $d(x,(g \circ f)(x)) \leq C$ for all $x \in X$ and $d^{\prime}\left(x^{\prime},(f \circ g)\left(x^{\prime}\right)\right) \leq C$ for all $x^{\prime} \in X^{\prime}$. It is said that $f$ and $g$ are quasi-inverses of each other with constant $C$. So, a quasi-isometric embedding $f$ is an quasi-isometry if and only if $f$ has a quasi-inverse $g$. 


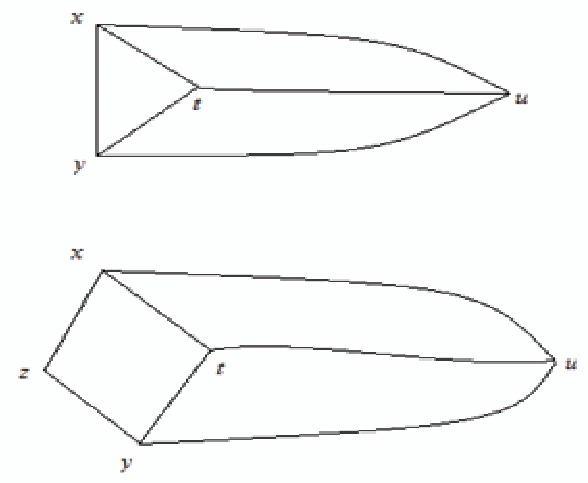

Figure 1. Triangle condition and quadangle condition.

We may assume that $f$ and $g$ are $(\lambda, \varepsilon)$-quasi-isometries with the same $\lambda$ and $\varepsilon$.

The next lemma shows a way that a geometrical action and quasi-isometry are connected. For a proof of this lemma, see Proposition 8.19 in [1].

Lemma 2.2 (Švarc-Milnor). If a group $G$ acts properly, cocompactly, and by isometries on a length space $X$, then $G$ is finitely generated and, with any base point $x_{0} \in X$, the mapping $g \mapsto g \cdot x_{0}$ is a quasi-isometry.

A Cayley graph is an examplary space that a group acts geometrically on and the group is quasi-isometric into. Let $A$ be a finite generating set for a group $G$. The vertex set of the Cayley graph $\Gamma(G, A)$ is $G$. The edge set is $G \times A$, the set of all edges $(g, a)$ from $g$ to $g a$ labeled by $a$. We regard the group itself as a metric space. Then, the inclusion $G \hookrightarrow \Gamma(G, A)$ by $g \mapsto g \cdot 1$ is a quasi-isometry with $\lambda=1$, $\varepsilon=0$, and $C=1 / 2$. However, Cayley graphs of a group vary according to the group presentation. In other words, letting $B$ be another finite generating set for $G$, the Cayley graphs $\Gamma(G, A)$ and $\Gamma(G, B)$ are different but quasi-isometric of each other.

2.4. Median graphs and median complexes. A graph $\Gamma$ is weakly modular if $\Gamma$ has the two conditions stated below (see Figure 1):

- Triangle Condition: for any three vertices $x, y, u$ with $1=d(x, y)<d(x, u)=$ $d(y, u)$, there exists a common neighbor $t$ of $x$ and $y$ such that $d(t, u)=$ $d(x, u)-1$.

- Quadrangle Condition: for any four vertices $x, y, z, u$ with $d(x, z)=d(y, z)=$ 1 and $d(x, u)=d(y, u)=d(z, u)-1$, there exists a common neighbor $t$ of $x$ and $y$ such that $d(t, u)=d(x, u)-1$.

A weakly modular graph $\Gamma$ becomes a modular graph if and only if $\Gamma$ is trianglefree and satisfies quadangle condition. Equivalently, $\Gamma$ is modular if $I[x, y] \cap I[y, z] \cap$ 
$I[z, x]$ is nonempty for any triplet $x, y, z \in V(\Gamma)$. The set $I[x, y] \cap I[y, z] \cap I[z, x]$ is called a median for $x, y, z$ and denoted by $m(x, y, z)$. A graph $\Gamma$ is a median graph if $m(x, y, z)$ is a singlton. Median structures are intimately related to hypercubes. See the next proposition; detailed discussion is found in [2].

Proposition 2.3 (Bandelt, 1984). Median graphs are exactly the retracts of hypercubes. Every median graph with more than two vertices is either a Cartesian product or a gated amalgam of proper median subgraphs.

Every finite median graph can be obtained by successive application of gated amalgamations of hypercubes. A median graph $\Gamma$ gives rise to an abstract cubical complex $K(\Gamma)$, called median complexes, consisting of cubes of any dimensions. Also conversely, $\Gamma$ is recovered from its complex $|K(\Gamma)|$ as the underlying graph. Thus, $\mathrm{CAT}(0)$ cubical complexes and median complexes are regarded as the same; the detailed discussion is shown in [2]. We define the n-median complex as the geometric realization of the CAT(0) cubical complex whose cubes are at most $n$-dimensional.

\section{L(0) SPACES AND L(0) GROUPS}

The $\mathrm{L}(\delta)$ property was well discussed in [3] where the notation $L_{\delta}$ was used for $\mathrm{L}(\delta)$. Let $X$ be a geodesic metric space with the metric $d$. A finite sequence of points $x_{1}, x_{2}, \ldots, x_{n}$ in $X$ constitutes a $\delta$-path if there exists a non-negative constant $\delta$ such that

$$
d\left(x_{1}, x_{2}\right)+d\left(x_{2}, x_{3}\right)+\ldots+d\left(x_{n-1}, x_{n}\right) \leq d\left(x_{1}, x_{n}\right)+\delta .
$$

Note that a $\delta$-path for $\delta=0$ is a geodesic in $X$. Let $\Delta\left(x_{1}, x_{2}, x_{3}\right)$ be a triangle in $X$. A point $t \in X$ is called $\delta$-center for $\Delta$ if $\left(x_{i}, t, x_{j}\right)$ is a $\delta$-path for the geodesic side $\left[x_{i}, x_{j}\right]$ where $i, j \in\{1,2,3\}$ and $j \equiv i+1 \bmod 3$. A geodesic space $X$ becomes a $\mathrm{L}(\delta)$ space if every trianlge in $X$ has a $\delta$-center. A group $G$ is an $L(\delta)$ group if it acts geometrically on an $\mathrm{L}(\delta)$ space for some $\delta \geq 0$.

A geodesic space $X$ is to be an $\mathrm{L}(0)$ space if the required $\delta$ value is zero, that is, every triangle $\Delta\left(x_{1}, x_{2}, x_{3}\right)$ in $X$ has a 0 -center $t$, say. Then, $\left(x_{i}, t, x_{j}\right)$ are all geodesics (0-paths); that is,

$$
t \in I\left[x_{1}, x_{2}\right] \cap I\left[x_{2}, x_{3}\right] \cap I\left[x_{3}, x_{1}\right]=m\left(x_{1}, x_{2}, x_{3}\right) \subset X .
$$

It may be said that $\mathrm{L}(0)$ space is a median space if the median of each geodesic triangle is unique.

Definition 3.1 (L(0) group). A finitely generated group $G$ is said to be an $\mathrm{L}(0)$ group if it acts properly, cocompatly, and by isometries on an $\mathrm{L}(0)$ space. 
If the $\mathrm{L}(0)$ property is an quasi-isometry invariant, then we can define an $\mathrm{L}(0)$ group on its Cayley graph. Let $X$ and $X^{\prime}$ be L(0) spaces with the metrics $d$ and $d^{\prime}$, respectively. Suppose $f: X \rightarrow X^{\prime}$ and $g: X^{\prime} \rightarrow X$ are $(\lambda, \varepsilon, C)$-quasi-inverses such that if $t \in m(x, y, z) \subset X$, then $t \mapsto t^{f} \in m\left(x^{f}, y^{f}, z^{f}\right)$. Then,

$$
\begin{aligned}
d(x, t)+d(t, y) & \leq \lambda\left(d^{\prime}\left(x^{f}, t^{f}\right)+d^{\prime}\left(t^{f}, y^{f}\right)\right)+2 \varepsilon \\
& \leq \lambda d^{\prime}\left(x^{f}, y^{f}\right)+2 \varepsilon \\
& \leq \lambda\left[\lambda\left(d\left(x^{f g}, x\right)+d(x, y)+d\left(y, y^{f g}\right)\right)+\varepsilon\right]+2 \varepsilon \\
& \leq \lambda^{2} d(x, y)+2 \lambda C+\lambda \varepsilon+2 \varepsilon
\end{aligned}
$$

As shown in the inequalities above, the $\mathrm{L}(0)$ property is not a quasi-isometry invariant but is preserved by an isometry. Weighting on generating set for a group perphaps would be an idea for preserving the $\mathrm{L}(0)$ property beteen two Cayley graphs. For example, both $\langle a, b \mid a b=b a\rangle$ and $\langle a, b, c \mid a b=c, b a=c\rangle$ are presentations of $\mathbb{Z}^{2}$. However, $\langle a, b \mid a b=b a\rangle$ is an $\mathrm{L}(0)$ group but $\langle a, b, c \mid a b=c, b a=c\rangle$ is not. If we assign the weight on $c$ by $\omega(c)=\omega(a)+\omega(b)=1+1=2$, then $\Gamma\left(\mathbb{Z}^{2},\{a, b, c\}\right)$ is also an $\mathrm{L}(0)$ space. This is the process of cubulating spaces introduced in [11].

The L(0) property can be considered on a CAT(0) cubical complex in the sense that the 1-skeleton of the cubical complex is a median graph. But the 1-skeletons of triangular or hexagonal complexes are not. By the Propositions 2.3 and the fact that $\mathrm{CAT}(0)$ cubical complexes and median complexes are the same, the next proposition is obtained. See [1] for detailed proof.

Proposition 3.2 (Chatterji and Ruane, 2002). The 0-skeleton of a CAT(0) cubical complex, endowed with the distance of the 1-skeleton, is an $L(0)$ space.

\section{Main Results}

Free groups are hyperbolic groups [7] and hyperbolic groups are very strong $L_{\delta}$ groups [4], which is renamed as word $\mathrm{L}(\delta)$ groups in the paper. Thus, free groups are word $\mathrm{L}(\delta)$ groups. More precisely, free groups acts on median graphs.

Lemma 4.1. A free group with a finite rank acts geometrically on a median graph.

Proof. Let $G$ be a free group of a fintie rank and let $A$ be a finitely inverse-closed generating set for $G$. The Cayley graph $\Gamma(G, A)$ is a tree so a unique geodesic space. We show that $\Gamma(G, A)$ is a median graph. Choose $x, y, z$ in $G=V(\Gamma(G, A))$. Figure 2 shows the simplification of image of $x, y$ and $z$ in $\Gamma(G, A)$. 


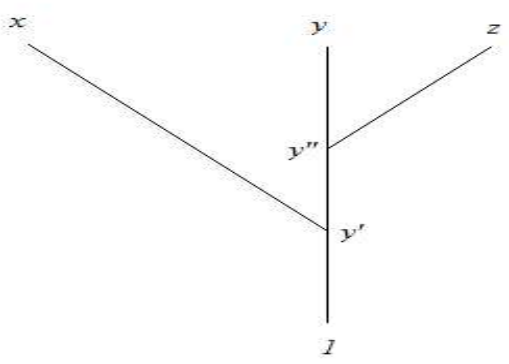

Figure 2. Free group presentation.

There exist two point $y^{\prime}$ and $y^{\prime \prime}$ in $I[1, y]$ such that they are gates for $x$ and $z$, respectively. Without loss of generality, assume that

$$
y^{\prime} \leq y^{\prime \prime} \leq y
$$

where $\leq$ is the order in $I[1, y]$. Thus,

$$
m(x, y, z)=I[y, x] \cap I[y, z] \cap I[x, z]=\left\{y^{\prime \prime}\right\} .
$$

So, a median $y^{\prime \prime}$ is a 0 -center for the triplet $x, y, z$. Note that the existance of a median $y^{\prime \prime}$ guarantees that $\Gamma(G, A)$ is a modular graph, so $\mathrm{L}(0)$ space. It is enough to say that free groups are L(0) groups.

We now verify the uniqueness of the median to show that free groups are groups acting on median graphs. Suppose $m=m(x, y, z)=l$ and $m \neq l$. Then there are at least two geodsics from $y$ to $x, y$ to $z$, and $x$ to $z$. It imediately implies that there is a loop like $(x, m, y, l, x)$ in $\Gamma(G, A)$. This contradicts the fact that $\Gamma(G, A)$ is a tree. So, $y^{\prime \prime} \in \Gamma(G, A)$ is the unique zero-center for the geodesic triplet $x, y, z$, and therefore, the Cayley graph $\Gamma(G, A)$ is a median graph.

Recall that $|\Gamma|$ is a geometric realization of an abstract graph $\Gamma=(V, E)$. If $V$ with the shortest path metric is an $\mathrm{L}(0)$ space, then $|\Gamma|$ is an $\mathrm{L}(\delta)$ space for some $\delta>0$. Let $G$ be an $\mathrm{L}(0)$ group and $d_{A}$ be a word metric induced from a finite generating set $A$ for $G$. Then, $\left(G, d_{A}\right)$ is a $\mathrm{L}(0)$ space and it is quasi-isometric to $\left(\Gamma(G, A), d_{A}\right)$ with quasi-isometry constant $\frac{1}{2}$. It implies that $\Gamma(G, A)$ is an $\mathrm{L}(\delta)$ space for $\delta=\frac{1}{2}$.

Let $X$ be an $n$-median complex and $X^{(1)}$ be the 1-skeleton of $X$, and let $x, y$ belong to different cubes in $X$. A metric $d$ on $X$ measures the distance between $x$ and $y$ along the parallel with $X^{(1)}$. Then, the distance between $x$ and $y$ is at most $\frac{n}{2}$. Consider a retraction $r: X \rightarrow X^{(1)}$ by which points from the central point of each cube correspond to a point in the nearest edge. By the triangle inequalities 
below:

$$
\begin{aligned}
d(x, y) & \leq d\left(x, x^{\prime}\right)+d\left(x^{\prime}, y^{\prime}\right)+d\left(y^{\prime}, y\right) \\
& \leq d\left(x^{\prime}, y^{\prime}\right)+n \\
d\left(x^{\prime}, y^{\prime}\right) & \leq d\left(x^{\prime}, x\right)+d(x, y)+d\left(y, y^{\prime}\right) \\
& \leq d(x, y)+n,
\end{aligned}
$$

a quasi-isometry inequality $d(x, y)-n \leq d\left(x^{\prime}, y^{\prime}\right) \leq d(x, y)+n$ is induced. So, $r$ is a quasi-isometric embedding. Also, since the retraction is a continuous surjective mapping into a subspace, a quasi-isometry constant for $r$ is zero. We use this retraction $r: X \rightarrow X^{(1)}$ to discuss the $\mathrm{L}(\delta)$ property on a median complex $X$ in the next theorem.

Theorem 4.2. Finitely generated groups acting geometically on n-median complexes are $L(2 n)$ groups.

Proof. Let $G$ be a finitely generated group and let $X$ be an $n$-median complex which $G$ acts geometically on. Choose $x_{1}, x_{2}, x_{3}$ arbiturary in $X$. Then, there exist $x_{i}^{\prime} \in X^{(1)}$ for $i=1,2,3$ such that $x_{i}^{\prime}$ is the nearest point from $x_{i}$ respectively. Note that $d\left(x_{i}, x_{i}^{\prime}\right)$ is at most $\frac{n}{2}$. Because $X^{(1)}$ is a median graph, there exists a median $t \in m\left(x_{1}, x_{2}, x_{3}\right)$ so that $d\left(x_{i}^{\prime}, t\right)+d\left(t, x_{j}^{\prime}\right)=d\left(x_{i}^{\prime}, x_{j}^{\prime}\right)$ for $i, j \in\{1,2,3\}$ and $j \equiv i+1$ mod 3.Thus, $t$ is a 0 -center for $\Delta\left(x_{1}^{\prime}, x_{2}^{\prime}, x_{3}^{\prime}\right)$.

Now show that $t$ is a $\delta$-center for $\Delta\left(x_{1}, x_{2}, x_{3}\right)$ for some $\delta$. From the triangle inequalities below:

$$
\begin{aligned}
d\left(x_{i}, t\right)+d\left(t, x_{j}\right) & \leq d\left(x_{i}, x_{i}^{\prime}\right)+d\left(x_{i}^{\prime}, t\right)+d\left(t, x_{j}^{\prime}\right)+d\left(x_{j}^{\prime}, x_{j}\right) \\
& \leq \frac{n}{2}+d\left(x_{i}^{\prime}, x_{j}^{\prime}\right)+\frac{n}{2} \\
& \leq d\left(x_{i}^{\prime}, x_{i}\right)+d\left(x_{i}, x_{j}\right)+d\left(x_{j}, x_{j}^{\prime}\right)+n \\
& \leq d\left(x_{i}, x_{j}\right)+2 n,
\end{aligned}
$$

where $i, j \in\{1,2,3\}$ and $j \equiv i+1 \bmod 3, t$ is a $2 n$-center of a geodesic triangle $\Delta\left(x_{1}^{\prime}, x_{2}^{\prime}, x_{3}^{\prime}\right)$. Therefore, $X$ is an $\mathrm{L}(2 n)$ space and so $G$ is an $\mathrm{L}(2 n)$ group.

Corson and Ryang [3] showed that L(0) groups are closed under taking direct product. A group acting on a median graph is an $\mathrm{L}(0)$ group so groups acting on median graphs are also closed under taking direct product. A group acting on an $n$-median complex is an $\mathrm{L}(2 n)$ group (Theorem 4.2). As a corrollary, the direct product of two $\mathrm{L}(2 n)$ groups becomes an $\mathrm{L}(4 n)$ group by the same argument in Corson and Ryang [3]. 


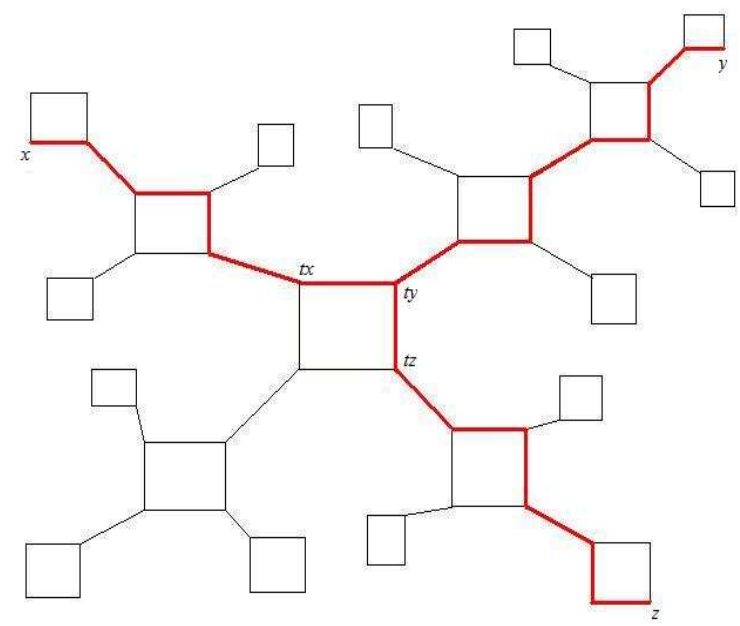

Figure 3. Gated amalgam of $C_{2}$ and $C_{4}$.

We then turn to the free product of groups acting on median graphs. First, look at a specific case. The cyclic group $C_{2}$ of order 2 and the the cyclic group of order 4 are L(0) groups because a Cayley graph of $C_{2}$ is the (underlying graph) of a 1median complex and a Cayley graph of $C_{4}$ is the underlying graph of a 2-median complex. The next Lemma discusses the free product of $C_{2}$ and $C_{4}$ as being an $\mathrm{L}(0)$ group.

Lemma 4.3. $C_{2} * C_{4}$ is a group acting on a median graph.

Proof. Let $K$ be a 1-dimensional cube and $K \times K$ a 2-dimensional cube. Note that $C_{2} \cong\left\langle a \mid a^{2}=1\right\rangle$ acts on $\Gamma(K)=\left(K^{(0)}, K^{(1)}\right)$ and $C_{4} \cong\left\langle b \mid b^{4}=1\right\rangle \operatorname{acts}$ on $\Gamma(K \times$ $K)=\left((K \times K)^{(0)},(K \times K)^{(1)}\right)$. Regard $\left(K^{(0)}, K^{(1)}\right)$ as the Cayley graph $\Gamma\left(C_{2}, a\right)$ and $\left((K \times K)^{(0)},(K \times K)^{(1)}\right)$ as the Cayley graph of $\Gamma\left(C_{4}, b\right)$. Then, consider the gated amalgam $\Gamma$ of $\Gamma(K)$ and $\Gamma(K \times K)$ as seen in Figure 3 which $C_{2} * C_{4}$ acts on. Choose $x, y, z$ in $\Gamma$. Then, each geodesic $[x, y],[y, z]$, and $[z, x]$ are common in one point $t_{y}$, in this case. The vertex $t_{y}$ is actually the unique median of $t_{x}, t_{y}, t_{z}$ in the loop which is a copy of $C_{4}$.

Theorem 4.4. Let $G_{1}$ and $G_{2}$ be word $L(0)$ groups. Then $G=G_{1} * G_{2}$ is a word L(0) group.

Proof. Let $G_{1}$ and $G_{2}$ be goups with a standard generating sets, respectively. Then, construct a Cayley graph $\Gamma(G)$ so that each vertex of $\Gamma\left(G_{i}\right)$ have $\Gamma\left(G_{j}\right)$ with $1_{G_{j}}$ on the vertex where $i, j \in\{1,2\}$ and $j \equiv i+1 \bmod 2$. Consider a retraction $\phi$ which degenerates all loops in $\Gamma$. Note then that $\Gamma_{0}=\phi(\Gamma)$ is a loop-free. If $[\mathrm{x}, \mathrm{y}]$ is a geodesic in $\Gamma$, then $\phi[\mathrm{x}, \mathrm{y}]$ is also a geodesic in $\Gamma_{0}$ since a geodesic in $\Gamma$ is shortened 
by $\phi$ in a unique way.

Choose $x, y, z$ in $\Gamma$. Then the loop including $x$ (respectively, $y$ and $z$ ) retracts into a point $x_{0}$ (respectively, $y_{0}$ and $z_{0}$ ). The loop is possibly trivial. For geodesics $[x, y]$, $[y, z]$, and $[z, x]$ in $\Gamma, \phi[x, y]=\left[x_{0}, y_{0}\right], \phi[y, z]=\left[y_{0}, z_{0}\right]$, and $\phi[z, x]=\left[z_{0}, x_{0}\right]$ are also geodesics in $\Gamma_{0}$. Since $\Gamma_{0}$ is a median graph, there exists a point $t_{0} \in m\left(x_{0}, y_{0}, z_{0}\right)$ in $\Gamma_{0}$, and thus $t_{0}$ becomes the 0 -center of the triplet $x_{0}, y_{0}, z_{0}$. The pre-image of $t_{0}$ is a loop in $\Gamma$; let $t_{x}, t_{y}$, and $t_{z}$ in the loop be the gate for $x, y$, and $z$, respectively. Then, $t_{x}, t_{y}, t_{z}$ are in the same copy of a free factor, say $G_{1}$ without loss of generality. Since $G_{1}$ is an $\mathrm{L}(0)$ group, there exists a 0 -center $t$ for the triplet $t_{x}, t_{y}, t_{z}$ in a copy $\bar{G}_{1}$ of $G_{1}$, i.e.,

$$
t \in m\left(t_{x}, t_{y}, t_{z}\right) \subset \bar{G}_{1} .
$$

Claim that $t \in m(x, y, z)$. Noting that $x^{\prime}, t_{x}, t_{y}, y^{\prime} \in[x, y]$, the geodesic $[x, y]$ is broken down by the partition of:

$$
I[x, y]=I\left[x, x^{\prime}\right] \cup\left[x^{\prime}, t_{x}\right] \cup\left[t_{x}, t_{y}\right] \cup\left[t_{y}, y^{\prime}\right] \cup\left[y^{\prime}, y\right] .
$$

Also, $y^{\prime}, t_{y}, t_{z}, z^{\prime} \in[y, z]$, and $x^{\prime}, t_{x}, t_{z}, z^{\prime} \in[x, z]$, the deodesics $[y, z]$ and $[z, x]$ are also broken down by the partitions in the same fashion. In the partitions, apparently the sub-geodesics in the right hand side are disjoint except $\left[t_{x}, t_{y}\right],\left[t_{y}, t_{z}\right]$, and $\left[t_{z}, t_{x}\right]$. Then, by definition,

$$
\begin{aligned}
t & \in m\left(t_{x}, t_{y}, t_{z}\right) \\
& =I\left[t_{x}, t_{y}\right] \cap I\left[t_{y}, t_{z}\right] \cap I\left[t_{z}, t_{x}\right] \\
& \subset I[x, y] \cap I[y, z] \cap I[z, x] \\
& =m(x, y, z) .
\end{aligned}
$$

So, a triplet $x, y, z$ in $\Gamma$ has a zero-center $t$, and therefore, $\Gamma$ is an $\mathrm{L}(0)$ space so $G$ is an $\mathrm{L}(0)$ group.

\section{FURTher StUdies}

Word hyperbolic groups associated with Cayley graphs are not different from (general) hyperbolic groups associated with hyperbolic spaces because the hyperbolicity is a quasi-isometry invariant between two geodesic spaces. However, the $\mathrm{L}(\delta)$ property is not quasi-isometry invariant so there is no guarantee that word $\mathrm{L}(\delta)$ groups and $\mathrm{L}(\delta)$ groups are the same. Mathematicians found some some similarities between the word- $\mathrm{L}(\delta)$ group and the $\mathrm{L}(\delta)$ group. For example, both groups 
are closed under taking direct product [3]; both have the same isoperimetric functions $[4,5]$. It is still an open problem to determine whether of not the two groups are the same.

Open problem. [CBMS Geometric Group Theory Confernce, Albany, NY, 2004] If a finitely generated group $G$ acts geometrically on an $\mathrm{L}(\delta)$ space, then is there a (weighted) generating set $A$ for $G$ such that $\Gamma(G, A)$ has the $\mathrm{L}(\delta)$ property?

We may consider this open problem in the case of $\delta=0$. Median complexes are the geometric realization of L $(\delta)$ groups. Especially, L $(0)$ groups act geometically on $\mathrm{L}(0)$ spaces which are loop-free 1-skeletons of median complexes. Using the median property, we discussed that a free product of word L(0) groups is a word L(0) group. Then, How about for the (general) L(0) groups? More characterization of $\mathrm{L}(0)$ and word $\mathrm{L}(0)$ groups will be expected in a furture research.

\section{REFERENCES}

1. M. Bridson \& A. Haefliger: Metric Spaces of Non-Positive Curvature. Springer-Verlag, Birlin, 1999.

2. V. Chepoi: Grpahs of some CAT(0) complexes. Advanced Applied Mathematics 24 (2000), 125-179.

3. J. Corson \& D. Ryang: Direct product of L-delta groups. Pure and Applied Mathematics 13 (2006), 269-280.

4. _ : Isoperimetric functions of groups acting on $\mathrm{L}(\delta)$ spaces. Glasgow Math. J. 49 (2007), 1-6.

5. M. Elder: $\mathrm{L}_{\delta}$ groups are almost convex and have a sub-cubic dehn function. Algebraic and Geometric Topology 4 (2004), 23-29.

6. M. Gromov: Hyperbolic groups. In S. M. Gersten, editor, Essays in Group Theory, pages 75-263. Springer-Verlag, 1987. Mathematical Science Research Institute Publications 8.

7. M. Neumanann \& M. Shapiro: A short course in geometric group theory. Notes for the Australia National University Workshops, 1996

8. G. Niblo \& L. Reeves: Groups acting on CAT(0) cube complexes. Geometry and Topology 1 (1997), 1-7.

9. _ : The geometry of cube complexes and the complexity of their fundamental groups. Topology 37 (1998), 621-633.

10. _ C Coxeter groups acts on CAT(0) cube complexes. J. Group Theory 6 (2003), 399-413.

11. B. Nica: Cubulating spaces with walls. Algebraic and Geometric Topology 4 (2004), 297-309. 
12. M. Sageev: Ends of group pairs ans non-positively curved cube complexes. Proc. London Math. Soc. 71 (1995), 585-671.

The University of North Carolina at Greensboro, NC 27402, USA

Email address: dryang@uncg.edu 